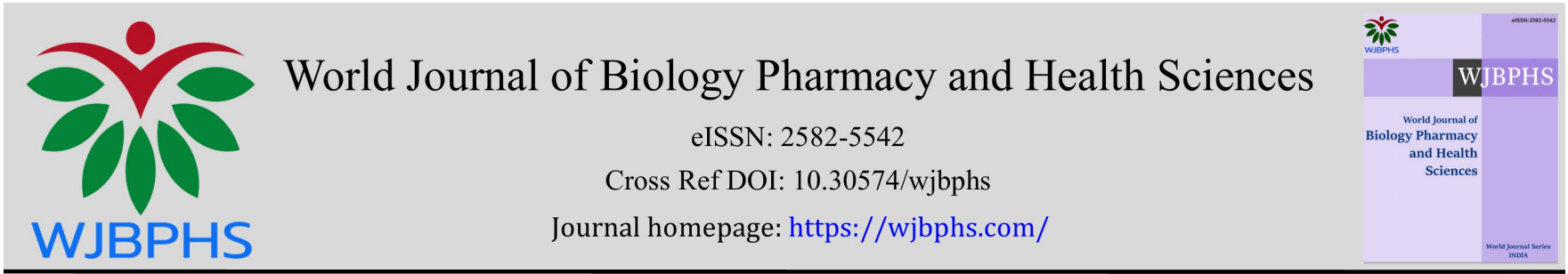

(RESEARCH ARTiCLE)

\title{
Effect of consanguinity on birth weight and birth defects in the Agadir region of Morocco
}

\author{
Karim Bouadil ${ }^{1}$ and Saïd El Madidi 2,* \\ ${ }^{1}$ Laboratory Biotechnologies and Valorization of Natural Resources (BVRN), Faculty of Sciences, University Ibn Zohr, \\ Agadir, Morocco. \\ 2 Biometrics and BioResources, Laboratory Biotechnologies and Valorization of Natural Resources (BVRN), Faculty of \\ Sciences, University Ibn Zohr, Agadir, Morocco.
}

World Journal of Biology Pharmacy and Health Sciences, 2022, 09(02), 055-059

Publication history: Received on 25 November 2021; revised on 08 January 2022; accepted on 10 January 2022

Article DOI: https://doi.org/10.30574/wjbphs.2022.9.2.0125

\begin{abstract}
The material of this study consists of 3701 newborns in the neonatology section, Maternity Hassan II Hospital, Agadir, Morocco from April 2016 to April 2018. For each newborn, several variables were recorded (birth weight, sex, consanguinity, gestational age, economic and social situation of parents, etc.). They types of birth defects have been classified according to the International Classification of Diseases (ICD-10). Of the 3701 newborns, 171 are carriers of congenital anomalies. This represents a prevalence of $4.6 \%$ (95\% CI: 3.9\% - 5.3\%). The prevalence of LBW was $8.7 \%$ (95\% CI: 7.7\% - 9.6\%). Of all newborns, 715 (19,64\%) were from consanguineous marriages and the mean inbreeding coefficient for the present study was 0.012 . The prevalence of congenital malformations were more frequently observed in consanguineous (7.69\%) compared to non-consanguineous (3.76\%). A significant association with consanguinity was observed for the congenital anomaly ( $p$-value $=0.00052$ ) and for LBW ( $p$-value $=0.0008$ ). Consanguinity was identified as significant risk factors associated with LBW and CA in this study.
\end{abstract}

Keywords: Birth defects; LBW; Consanguinity; Agadir; Morocco

\section{Introduction}

According to the World Health Organization, approximately 76,000 newborn babies die each year before the age of 28 days from birth defects [1]. These anomalies result from a pathological process during embryonic development, following a genetic (intrinsic) predisposition or the exposure of an extrinsic risk factor or a combination of both [1,2]. The prevalence of major CMs is around 3.0\% of live births [3,4]. Global surveys have shown that the prevalence of birth defects varies considerably from country to country. A wide variation in this prevalence is observed worldwide and ranges from less than $1.0 \%$ to more than $8.0 \%$.

The global incidence of LBW is around 17\%, although estimates vary from 19\% in the developing countries (countries where it is an important public health problem) to 5-7\% in the developed countries [5]. The estimated global prevalence of LBW in 2015 was 14.6\% (uncertainty interval: 12.4 -17.1) and that 20.5 million (17.4 to 24.0 million) live births were to low birth weight children, $91 \%$ in low income countries and intermediate, mainly South Asia (48\%) and sub-Saharan Africa (24\%) [6]. Several studies have analyzed the relationship between these major newborn health problems and the degree of consanguinity. Some studies have shown a significant association between inbreeding and CA [7,8] and LBW $[9,10]$.

\footnotetext{
${ }^{*}$ Corresponding author: El Madidi Saïd

Biometrics and BioResources, Laboratory Biotechnologies and Valorization of Natural Resources (BVRN), Faculty of Sciences, University Ibn Zohr, Agadir, Morocco. 
The main objective of this study is to analyze the effect of inbreeding as a risk factor for CA and LBW in the region of Agadir in Morocco. In Morocco, only a few studies exist concerning the association between birth defects and consanguinity. This work constitutes a contribution to a better understanding of these risk factors which are involved in the incidence of congenital anomalies an LBW in Morocco and in the region concerned by the study.

\section{Material and methods}

\subsection{Data collection}

This is a case-control study which was carried out in the pediatrics and neonatology department of the Hassan II hospital in Agadir. The study population consists of 3701 newborns admitted to the neonatology service from April 2016 to April 2018. The team of this study worked in the neonatal service of the hospital 3 days a week and the newborns included in this study are those whose parents cooperated voluntarily and fully to answer the various questions of the questionnaire survey. For each newborn, a questionnaire is completed including information relating to biological, economic and social factors, including maternal age, place of residence, educational level and socio-economic level. The types of birth defects have been classified according to the International Classification of Diseases (ICD-10) codes (5). The diagnosis of congenital anomalies is based on the clinical evaluation of the newborn by the pediatrician and other appropriate investigation methods such as radiography, ultrasound, echocardiography, etc.

\subsection{Consanguinity data}

Families were considered to be consanguineous if there was intermarriage between first cousins or other close relatives. In clinical genetics a consanguineous marriage is most commonly defined as a union between a couple related as second cousins or closer, equivalent to an inbreeding coefficient in their progeny of $F \geq 0.0156$ (15). The inbreeding coefficient (F) was calculated for each newborn and the mean $(\alpha)$ was estimated according to the formula

$$
\alpha=\sum \mathrm{f}_{\mathrm{i}} \mathrm{F}_{\mathrm{i}}
$$

where $\left(f_{i}\right)$ the proportion of each type of consanguineous relationship " $i$ ", and $\left(F_{i}\right)$ the corresponding inbreeding coefficient. Consanguineous marriages were classified by the degree of relatedness between couples: first cousins $(\mathrm{F}=$ $0.0625)$, double first cousins $(F=0.125)$, second cousins $(F=0.0156)$ and first cousin once removed or double second cousin $(F=0,0313)$.

\subsection{Statistical data analysis}

The data collected were first tabulated in Microsoft Excel and basic summer statistics were compiled from the data. For socio-demographic variables, the analyzes for significant associations were carried out using the chi-square test. Statistical analyzes were performed using IBM SPSS Statistics version 24 (IBM Corp) and R for Windows version 3.5 .3 (R Core Team).

\section{Results}

Figure 1, shows the geographical distribution of the mothers' places of residence included in this study. Of the 3640 families, $96 \%$ are domiciled in the Souss Massa region with $42 \%$ are from the city of Agadir and $47 \%$ from the city of Inezgane and the cities of Tiznit and Taroudant come in third place with a percentage of 3\%. 2088 (57.36\%) of the couples in the sample live in an urban area and 1,552 (42.64\%) live in rural areas.

Of all newborns, $715(19,64 \%)$ were from consanguineous marriages and the mean inbreeding coefficient ( $\alpha$ ) for the present study was 0.0119. The examination of data on the kinship of the newborns' parents revealed that for all consanguineous marriages, 611 (85\%) marriages were between first cousins, 64 (9\%) between second cousins and 29 ( $4 \%$ ) between double first cousins and the frequency of other marriages was very low (Table 1).

The sociodemographic characteristics of consanguineous and non consanguineous distribution in this study is shown in Table 2. Among the 3640 newborns, 1831 are males (50.31\%) and 1809 are females (49.69 \%) and no significant association was found between consanguinity and gender. A significant association was found between the degree of consanguinity and low educational level of the mother ( $p$-value $=0,020$ ) and the father ( $p$-value $=0,0017$ ). The difference between the proportion of consanguineous marriages in urban and rural areas was found to be highly significant. 1552 (42.64\%) families resided in rural areas and 2088 (57.36\%) in urban areas. The degree of consanguinity in rural areas was $22.4 \%$ against $17.58 \%$ in urban areas and the mean coefficient of consanguinity 
estimated in the rural and urban areas was $(0,01399)$ and $(0,0102)$ respectively. For mother's educational level, The proportion of consanguineous marriages among mothers with low educational level was $21.40 \%$ against $18.30 \%$ for mothers with a high or medium educational level. The same trend was observed for the father's educational level where a significantly higher proportion of consanguineous marriages is observed among fathers with a low level (23.23 \%) compared to a medium or high level $(18.56 \%)$.

Table 1 Frequency distribution of various types of consanguineous marriages

\begin{tabular}{|l|l|l|}
\hline Type of marriage & F & n \\
\hline First cousins & 0,0625 & 611 \\
\hline Second cousins & 0,0156 & 64 \\
\hline Double first cousins & 0,125 & 29 \\
\hline First cousins once removed & 0,03125 & 7 \\
\hline Double second cousins & 0,03125 & 4 \\
\hline Total consanguineous & & 715 \\
\hline Non consanguineous & & 2986 \\
\hline Total & & 3701 \\
\hline Mean coefficient of consanguinity & & 0,0119 \\
\hline
\end{tabular}

Table 2 Characteristics according newborn consanguinity

\begin{tabular}{|l|l|l|l|l|l|l|l|}
\hline Characteristics & & Frequency & Percent (\%) & C & NC & $\chi \mathbf{2}$ & p-value \\
\hline \multirow{3}{*}{ Gender } & Males & 1831 & 50.31 & 366 & 1465 & 0.279 & 0.587 \\
& Females & 1809 & 49.69 & 349 & 1460 & & \\
\hline \multirow{3}{*}{ Residence } & Rural & 1532 & 42,09 & 348 & 1204 & 13.24 & 0.0003 \\
& urban & 2108 & 57,91 & 367 & 1721 & & \\
\hline \multirow{5}{*}{ Fother's educational level } & low & 1575 & 43,27 & 337 & 1238 & 5.041 & 0.020 \\
& Medium & 1778 & 48,84 & 324 & 1454 & & \\
& High & 287 & 7,89 & 54 & 233 & & \\
\hline \multirow{5}{*}{ Family socio-economic level educational level } & low & 904 & 24,84 & 210 & 694 & 9.505 & 0,0017 \\
& Medium & 2151 & 59,09 & 402 & 1749 & & \\
& High & 585 & 16,07 & 103 & 482 & & \\
\hline \multirow{2}{*}{ LBW } & Medium & 1297 & 35,63 & 250 & 1047 & 1.650 & 0.438 \\
& High & 75 & 62,3 & 446 & 1822 & & \\
\hline CA & Yes & 320 & 8,64 & 78 & 242 & 5.75 & 0.0008 \\
& No & 3381 & 91,35 & 637 & 2744 & & \\
\hline & Yes & 171 & 4,62 & 55 & 116 & 18.09 & $<0.0001$ \\
& No & 3530 & 95,38 & 669 & 2861 & & \\
\hline
\end{tabular}

C: consanguineous, NC: non consanguineous, LBW: low birth weight, CA: Congenital anomalies. 


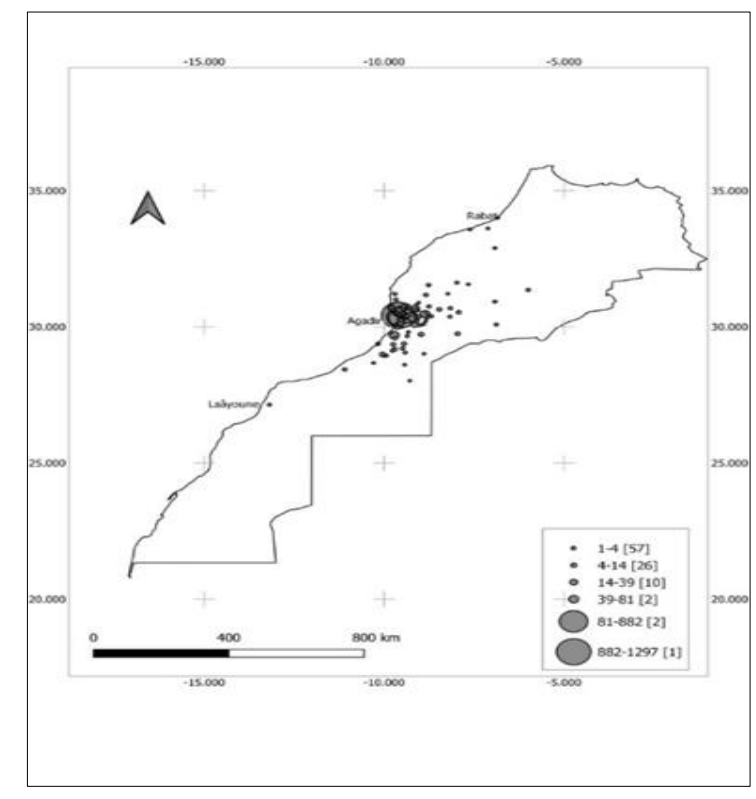

Figure 1 Geographic distribution of mothers' places of residence

\section{Discussion}

Our study also shows that consanguinity is very highly associated with the onset of birth defects. The level of consanguinity has been reported by several authors as a risk factor for congenital anomalies [11-13]. The level of consanguinity was highest among birth defects, and relatives were more likely to have infants with multiple malformations [11]. Shieh et al., Have shown that congenital heart disease is more frequent in consanguineous unions in the study population, mainly when the coefficient of consanguinity is greater than or equal to 0.0625 [12]. The prevalence of congenital anomalies has been observed mainly in consanguineous marriages compared to nonconsanguineous marriages [13]. In a population with a medium or high degree of consanguinity, the formulation of a public health program with a multi-approach strategy, including education on the genetic consequences of consanguineous marriages, prenatal diagnosis, neonatal screening and genetic counseling is a necessity to avoid an excessively high prevalence of congenital anomalies [14].

The prevalence of LBW in our study in the region of Agadir was 8.7\% (95\% CI: 7.7\% - 9.6\%). This value is close to that given by the WHO in 2004 for Moroccan newborns (11\%) [15]. This prevalence value is intermediate between that estimated at Mohammadia 5.3\% [16] and that reported in the region of Marrakech 12.31\% [17]. A significant statistical association between LBW and consanguinity. This result is confirmed by other works. Similarly, In Morocco, the risk of LBW was high among between related spouses [16]. In Jordan and in Saudi Arabia, consanguineous marriages were significantly associated with low-birth-weight delivery $[18,19]$. In others studies in Iran and Pakistan, consanguineous marriages, and especially between first cousins, have been shown to increase the risk of having a newborn with low birth weight $[20,21]$.

\section{Conclusion}

According to this study, the degree of consanguinity in this region was shown to be around $20 \%$. Consanguineous marriages between first cousins are the most common among other types of consanguineous unions. The reduction in the incidence of birth defects requires an improvement in the level of education with better social conditions and a better awareness of the risks of morbid diseases linked to consanguinity.

\section{Compliance with ethical standards}

\section{Acknowledgments}

Our sincere thanks go to all the members of the pediatric services of the Hassa II hospital (doctors and nurses) for their help and contributions to the realization of the study. 


\section{Disclosure of conflict of interest}

There are no conflicts of interest among the authors of this study.

\section{Statement of informed consent}

Only parents who gave their consent to freely answer all survey questions were included in this study.

\section{References}

[1] World Health Organization. Section on congenital anomalies. 2012.

[2] World Health Organization. Congenital anomalies Key facts. 2016.

[3] Anthony S, Kateman H, Brand R, et al. Ethnic differences in congenital malformations in the Netherlands: analyses of a 5-year birth cohort. Paediatr Perinat Epidemiol. 2005; 19: 135-44.

[4] Kalter H. Teratology in the 20th century: environmental causes of congenital malformations in human and how they were established. Neurotoxicol Teratol. 2003; 25: 131-282.

[5] De Bernabé J V, Soriano T, Albaladejo R, Juarranz M, Calle, ME, Martínez D, Domínguez-Rojas V. Risk factors for low birth weight: a review. European Journal of Obstetrics \& Gynecology and Reproductive Biology. 2004; 116(1): 3-15.

[6] Blencowe H Krasevec J de Onis M et al. National, regional, and worldwide estimates of low birthweight in 2015, with trends from 2000: a systematic analysis. Lancet Glob Health. 2019; (published online May 15.)

[7] Magnus P, Berg K, Bjerkedal T. Association of parental consanguinity with decreased birth weight and increased rate of early death and congenital malformations. Clinical genetics. 1985; 28(4): 335-342.

[8] Kulkarni ML, Kurian M. Consanguinity and its effect on fetal growth and development: a south Indian study. Journal of medical genetics. 1990; 27(6): 348-352.

[9] Bener A, Saleh NM, Salameh KM, Basha B, Joseph S, Al Buz R. Socio-demographic and consanguinity risk factors associated with low birthweight. J Pak Med Assoc.2013; 63(5): 598-603.

[10] Mumtaz G, Tamim H, Kanaan M, Khawaja M, Khogali M, Wakim G, Yunis KA. Effect of consanguinity on birth weight for gestational age in a developing country. American Journal of Epidemiology. 2007; 165(7): 742-752.

[11] Al-Gazali LI, Dawodu AH, Sabarinathan K, Varghese M. The profile of major congenital abnormalities in the United Arab Emirates (UAE) population. Journal of medical genetics. 1995; 32(1): 7-13.

[12] Shieh JTC, Bittles AH, Hudgins L. Consanguinity and the risk of congenital heart disease. Am J Med Genet. 2012; Part A 158 A: 1236-1241.

[13] Tayebi N, Yazdani K, Naghshin N. The prevalence of congenital malformations and its correlation with consanguineous marriages. Oman medical journal. 2010; 25(1): 37.

[14] Kanaan ZM, Mahfouz R, Tamim H. The prevalence of consanguineous marriages in an underserved area in Lebanon and its association with congenital anomalies. Genetic testing. 2008; 12(3): 367-372.

[15] Wardlaw Tessa M. Low Birth weight: Country, regional and global estimates. UNICEF. 2004.

[16] Hassoune S, Bassel S, Nani S, Maaroufi A. Prevalence and associated factors of low birth weight in the provincial hospital of Mohammedia--Morocco. La Tunisie Médicale. 2015; 93(7): 440-444.

[17] Elkhoudri N, Baali A. Prevalence and determinants of low birth weight: a case-control study in marrakesh (morocco). Iranian journal of public health. 2015; 44(3): 422.

[18] Obeidat BR, Khader YS, Amarin ZO, Kassawneh M, Al Omari M. Consanguinity and adverse pregnancy outcomes: the north of Jordan experience. Maternal and child health journal. 2010; 14(2): 283-289.

[19] Wong SS, Anokute CC. The effect of consanguinity on pregnancy outcome in Saudi Arabia. Journal of the Royal Society of Health. 1990; 110(4): 146-147.

[20] Chaman R, Taramsari MG, Khosravi A, Amiri M, Naieni KH, Yunesian M. Consanguinity and neonatal death: a nested case-control study. Journal of family \& reproductive health. 2014; 8(4): 189.

[21] Badshah S, Mason L, McKelvie K, Payne R, Lisboa PJ. Risk factors for low birthweight in the public-hospitals at Peshawar, NWFP-Pakistan. BMC public health. 2008; 8(1): 1-10. 